\title{
Cryptosporidium parvum
}

National Cancer Institute

\section{Source}

National Cancer Institute. Cryptosporidium parvum. NCI Thesaurus. Code C122276.

A species of parasitic protozoa in the family Cryptosporidiidae. C. parvum is one of the main agents of cryptosporidiosis. Transmission occurs mainly through contact with contaminated water and infection is generally self-limiting in immunocompetent individuals. 